\title{
First-principles study of van der Waals interactions and lattice mismatch at $\mathrm{MoS}_{2} /$ metal interfaces
}

\author{
Mojtaba Farmanbar" and Geert Brocks ${ }^{\dagger}$ \\ Faculty of Science and Technology and MESA ${ }^{+}$Institute for Nanotechnology, University of Twente, \\ P.O. Box 217, 7500 AE Enschede, The Netherlands
}

(Received 23 October 2015; revised manuscript received 13 January 2016; published 2 February 2016)

\begin{abstract}
We explore the adsorption of $\mathrm{MoS}_{2}$ on a range of metal substrates by means of first-principles density functional theory calculations. Including van der Waals forces in the density functional is essential to capture the interaction between $\mathrm{MoS}_{2}$ and a metal surface, and obtain reliable interface potential steps and Schottky barriers. Special care is taken to construct interface structures that have a mismatch between the $\mathrm{MoS}_{2}$ and the metal lattices of $<1 \%$. $\mathrm{MoS}_{2}$ is chemisorbed on the early transition metal Ti, which leads to a strong perturbation of its (electronic) structure and a pinning of the Fermi level $0.54 \mathrm{eV}$ below the $\mathrm{MoS}_{2}$ conduction band due to interface states. $\mathrm{MoS}_{2}$ is physisorbed on $\mathrm{Au}$, where the bonding hardly perturbs the electronic structure. The bonding of $\mathrm{MoS}_{2}$ on other metals lies between these two extreme cases, with interface interactions for the late $3 d$ transition metals $\mathrm{Co}, \mathrm{Ni}$, $\mathrm{Cu}$ and the simple metal $\mathrm{Mg}$ that are somewhat stronger than for the late $4 d / 5 d$ transition metals $\mathrm{Pd}, \mathrm{Ag}, \mathrm{Pt}$ and the simple metal Al. Even a weak interaction, such as in the case of Al, gives interface states, however, with energies inside the $\mathrm{MoS}_{2}$ band gap, which pin the Fermi level below the conduction band.
\end{abstract}

DOI: 10.1103/PhysRevB.93.085304

\section{INTRODUCTION}

Transition-metal dichalcogenides (TMDs) such as molybdenum disulfide $\left(\mathrm{MoS}_{2}\right)$ have layered structures, where the atoms within a TMD monolayer form a covalently bonded planar network, and the interaction between these layers is a weak, van der Waals interaction [1,2]. A monolayer of $\mathrm{MoS}_{2}$ consists of a layer of molybdenum atoms sandwiched between two layers of sulfur atoms. $\mathrm{MoS}_{2}$ monolayers can be exfoliated through micromechanical cleavage, similar to graphene or boron nitride [3]. Unlike graphene (a metal), or boron nitride (an insulator), $\mathrm{MoS}_{2}$ is a semiconductor. Moreover, whereas bulk $\mathrm{MoS}_{2}$ has an indirect band gap $(1.2 \mathrm{eV})$, monolayer $\mathrm{MoS}_{2}$ has a direct band gap $(\sim 1.8-1.9 \mathrm{eV})$, and shows a strong optical absorption and luminescence [4,5]. At present, $\mathrm{MoS}_{2}$, and TMDs in general, are vehemently pursued as promising materials for applications in electronics and optoelectronics $[5,6]$.

Contacting $\mathrm{MoS}_{2}$ to metal electrodes proves to be a problem; it tends to produce unexpectedly high interface resistances, indicative of a high Schottky barrier at the interface [7-12]. A high barrier could be caused by strong interface bonding creating interface states that pin the Fermi level [13] or by weak bonding creating a potential step due to Pauli repulsion at the interface $[14,15]$. The nature of the interaction at the $\mathrm{MoS}_{2} /$ metal interface is far from trivial. On the one hand, one could argue that, as $\mathrm{MoS}_{2}$ has no dangling bonds at its surface, its interaction with metal substrates should be weak and of van der Waals type. On the other hand, many metal species form (di)chalcogenide compounds [4,5,16,17], and when adsorbing $\mathrm{MoS}_{2}$ onto a metal substrate, there could be a competition between the metal surface and the Mo atoms for interacting with the sulfur atoms at the interface. In that case, not only the $\mathrm{MoS}_{2} /$ metal bonding would be a much stronger chemical bonding, but also the structure

\footnotetext{
*m.farmanbar@utwente.nl

†g.h.1.a.brocks@utwente.nl
}

and electronic structure of the $\mathrm{MoS}_{2}$ adsorbate could be significantly perturbed.

In this paper, we explore the adsorption of $\mathrm{MoS}_{2}$ on a variety of metal substrates by means of first-principles density functional theory (DFT) calculations, following up on work briefly reported in a short paper [13]. Previous DFT studies have concentrated foremost on the Schottky barrier formed at $\mathrm{MoS}_{2} /$ metal interfaces using the local density approximation (LDA) [18-23]. LDA gives a reasonable description of the adsorption of graphene and $h$-BN on metal surfaces, but such results cannot be generalized to other systems, as it is known that LDA often leads to an unrealistic overbinding $[14,15,24-30]$. Other studies have used a generalized gradient approximation (GGA) functional, such as PBE [31], which apparently works well for TMDs adsorbed on metals [13,32,33], although it generally gives bad results for weakly bonded systems $[34,35]$.

Here, we focus on the interface interaction and its implications for the structure and electronic structure of the $\mathrm{MoS}_{2}$ adsorbate and the Schottky barrier. We choose a wide range of metal substrates: the (111) surfaces of $\mathrm{Al}, \mathrm{Ni}, \mathrm{Cu}$, $\mathrm{Pd}, \mathrm{Ag}, \mathrm{Pt}$, and $\mathrm{Au}$, and the (0001) surfaces of $\mathrm{Mg}$, Ti, and $\mathrm{Co}$, which are expected to have a wide range of interaction strengths with the adsorbate. As the interface interaction can vary from weak (physisorption) to strong (chemisorption), it is a priori not clear which DFT functional describes such bonding. We test and compare results obtained with a van der Waals functional, designed to describe weak, van der Waals, interactions [36-38], to results obtained with GGA and LDA functionals, which are conventionally used to describe chemical bonding. We assess the importance of van der Waals interactions for the interface interaction, and evaluate its effect on the structure and electronic structure of the $\mathrm{MoS}_{2}$ adsorbant.

We consider the situation where a $\mathrm{MoS}_{2}$ layer is adsorbed as a whole on a metal substrate, making it more likely that the integrity of the $\mathrm{MoS}_{2}$ layer is preserved in the adsorption process. If the $\mathrm{MoS}_{2} /$ metal interaction is not too strong, and the $\mathrm{MoS}_{2}$ and metal surface lattices are not matched, the interface 
structure is likely to be incommensurable. In a supercell calculation, one is forced to approximate such a structure by a commensurable one. Previous calculations have used small supercells, where in some cases appreciable artificial strain is generated because of the mismatch between the $\mathrm{MoS}_{2}$ and the metal-surface lattices [19,21-23]. We apply a strategy for choosing supercells such that the artificial strain is minimal, and test the influence of strain on the electronic properties of the interface.

This paper is organized as follows. Section II describes the DFT calculations, comparing different functionals in Sec. II B and discussing the effect of lattice mismatch in Sec. II C. Results are discussed in Sec. III, with the metal/MoS interaction in Sec. III A and its effects on the interface potential step and the Schottky barrier in Sec. III B. Strong chemisorption is discussed in more detail in Sec. III C, and a summary and the conclusions are presented in Sec. IV.

\section{CALCULATIONS}

\section{A. Computational methods}

We calculate ground-state energies and optimize geometries at the density functional theory (DFT) level, using projector-augmented waves (PAWs) as implemented in the VASP code [39-42]. The plane-wave kinetic-energy cutoff is set at $400 \mathrm{eV}$. The surface Brillouin zone is integrated with the Methfessel-Paxton technique using a smearing parameter of $0.05 \mathrm{eV}$ [43] and a $k$-point sampling grid with a spacing of $0.01 \AA^{-1}$. The $\mathrm{MoS}_{2}$ /metal interface is modeled as a slab of four to six layers of metal atoms with one or two layers of $\mathrm{MoS}_{2}$ adsorbed on one side and a vacuum region of $\sim 12 \AA$. The in-plane supercell is chosen such as to minimize the mismatch between the $\mathrm{MoS}_{2}$ and metal lattices, which is discussed in more detail in Sec. II C. A dipole correction is applied to avoid spurious interactions between periodic images of the slab [44]. We allow the positions of the atoms to relax until the force on each atom is smaller than $0.01 \mathrm{eV} \AA^{-1}$, except for the bottom layer of metal atoms, whose positions are kept fixed. The electronic self-consistency criterion is set to $10^{-5} \mathrm{eV}$.

It is well known that commonly used DFT exchangecorrelation functionals, based upon LDA [18] or GGA [31], give decent descriptions of covalent and ionic bonding, but they may fail for weakly bonded systems, as such functionals do not contain a description of van der Waals interactions. For example, GGA functionals such as PW91 or PBE [31] do not capture the bonding between $h$-BN or graphene layers, nor that between $h$-BN or graphene and transition-metal (111) surfaces $[35,45]$. A priori we do not know how important van der Waals interactions are in the bonding between $\mathrm{MoS}_{2}$ and a metal surface. In Sec. II B, we compare results obtained using a van der Waals density functional (vdW-DF) [36-38], with results obtained with GGA and LDA functionals.

One way of visualizing bonding at a $\mathrm{MoS}_{2} /$ metal interface is by the electron density difference

$$
\Delta n(\mathbf{r})=n_{\mathrm{M} \mid \mathrm{MoS}_{2}}(\mathbf{r})-n_{\mathrm{M}}(\mathbf{r})-n_{\mathrm{MoS}_{2}}(\mathbf{r}),
$$

where $n_{\mathrm{M} \mid \mathrm{MoS}_{2}}(\mathbf{r}), n_{\mathrm{M}}(\mathbf{r})$, and $n_{\mathrm{MoS}_{2}}(\mathbf{r})$ are the electron densities of $\mathrm{MoS}_{2}$ adsorbed on the metal, of the metal surface, and of the free-standing $\mathrm{MoS}_{2}$, respectively. The system as a

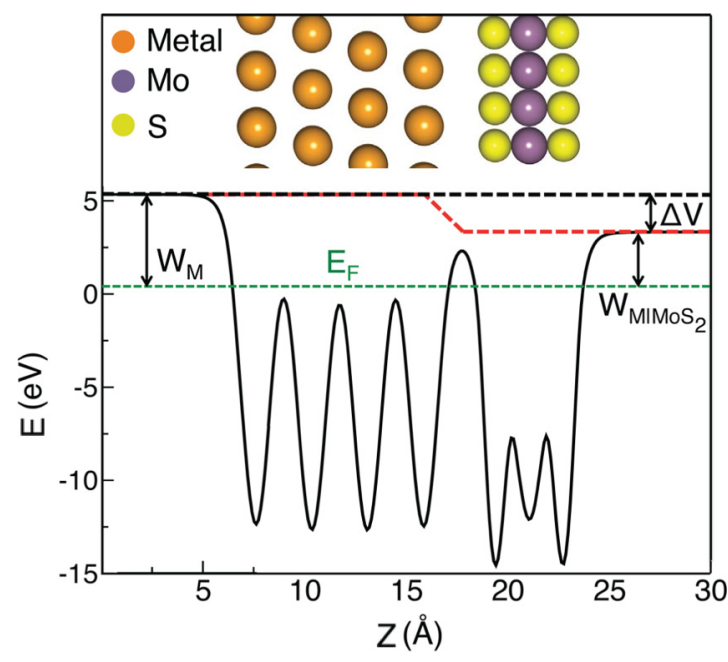

FIG. 1. Side view of metal/ $\mathrm{MoS}_{2}$ structure with corresponding plane-averaged electrostatic potential $\bar{V}(z)$ and $E_{F}$ the Fermi level. The interface potential step $\Delta V$ is reflected in the difference between the work function on the metal side $W_{\mathrm{M}}$ and on the $\mathrm{MoS}_{2}$ side $W_{\mathrm{M} \mid \mathrm{MoS}_{2}}$.

whole is neutral, and $\Delta n(\mathbf{r})$ is localized around the metal/MoS interface, i.e., $\Delta n(\mathbf{r}) \rightarrow 0$ for $\mathbf{r}$ sufficiently far from the interface. Solving the Poisson equation with $\Delta n(\mathbf{r})$ as source then gives a potential step across the interface

$$
\Delta V=\frac{e^{2}}{\epsilon_{0} A} \iiint z \Delta n(\mathbf{r}) d x d y d z .
$$

Here, $z$ is the direction normal to the interface, $A$ is the interface area, and $\Delta V$ is the difference between the asymptotic values of the potential left and right of the interface.

Figure 1 also illustrates an alternative expression for the potential step

$$
\Delta V=W_{\mathrm{M}}-W_{\mathrm{M} \mid \mathrm{MoS}_{2}},
$$

where $W_{\mathrm{M}}, W_{\mathrm{M} \mid \mathrm{MoS}_{2}}$ are the work functions of the clean metal surface, and of the metal surface covered by $\mathrm{MoS}_{2}$, respectively. A practical way of obtaining work functions from DFT calculations is to track the plane-averaged electrostatic (Hartree) potential $\bar{V}(z)$ into the vacuum (see Fig. 1), where typically the asymptotic value is reached with a few $\AA$ from the surface. In converged calculations, the expressions of Eqs. (2) and (3) give results that are with a few meV of one another.

The Schottky barrier height for electrons is defined as

$$
\Phi_{\mathrm{n}}=E_{F}-\chi_{\mathrm{MoS}_{2}},
$$

with $E_{F}$ the Fermi level and $\chi_{\mathrm{MoS}_{2}}$ the electron affinity of $\mathrm{MoS}_{2}$, both defined as distances to the vacuum level, i.e., positive numbers. There are several ways to extract the Schottky barrier height from $\mathrm{MoS}_{2} /$ metal slab calculations.

One could determine $\Phi_{\mathrm{n}}$ by measuring $E_{F}-\chi_{\mathrm{MoS}_{2}}$ in the band structure or in the density of states of the $\mathrm{MoS}_{2} /$ metal slab, as in Refs. [20-23]. In order to identify the $\mathrm{MoS}_{2}$ related states, one needs to calculate the amplitudes of the projections of the wave functions of the slab on the $\mathrm{MoS}_{2}$ layer. There is always some arbitrariness involved in such a projection if the adsorbate and the substrate are in close connection. In addition, identification of states belonging to the adsorbate is possible 
only if its electronic structure is not significantly perturbed in the adsorption process, which is only the case if the adsorbate is (weakly) physisorbed on the substrate [15]. In practice, we find that this procedure for obtaining the Schottky barrier height at $\mathrm{MoS}_{2}$ /metal interfaces is not sufficiently accurate when applied to the projected density of states, and of practical use only when applied to the projected band structure of a small supercell.

An alternative way of locating the conduction band edge $\chi_{\mathrm{MoS}_{2}}$ in a $\mathrm{MoS}_{2} /$ metal slab calculation, without having to resort to wave-function projections, is by aligning the core levels of the Mo or $\mathrm{S}$ atoms in the slab with the corresponding core levels in free-standing $\mathrm{MoS}_{2}$. It allows us to compare the densities of states of free-standing and adsorbed $\mathrm{MoS}_{2}$ (see Sec. III B), and, in principle, this procedure also allows for calculating the Schottky barrier height. Of course, this only makes sense if the $\mathrm{MoS}_{2}$ electronic structure is not perturbed too strongly by the adsorption.

Alternatively, the two quantities $E_{F}$ and $\chi_{\mathrm{MoS}_{2}}$ on the right-hand side of Eq. (4) are are easily obtained in separate calculations on an $\mathrm{MoS}_{2} /$ metal slab and a free-standing $\mathrm{MoS}_{2}$ layer, respectively. On the $\mathrm{MoS}_{2}$ side of the slab we have $E_{F}=W_{\mathrm{M} \mid \mathrm{MoS}_{2}}$ (see Fig. 1). Convergence as a function of slab thickness is usually faster if we use Eq. (3), and extract $\Delta V$ from a calculation on an $\mathrm{MoS}_{2}$ /metal slab and $W_{\mathrm{M}}$ from a separate calculation on a clean metal slab. The Schottky barrier height is then calculated as

$$
\Phi_{\mathrm{n}}=W_{\mathrm{M}}-\chi_{\mathrm{MoS}_{2}}-\Delta V .
$$

Of course, if the $\mathrm{MoS}_{2}$ electronic structure is very strongly perturbed by adsorption, one has to reconsider the definition of the Schottky barrier (see Sec. III C). In the following, the potential step $\Delta V$ is used to characterize the $\mathrm{MoS}_{2} /$ metal interface, along with the binding energy and the structure.

\section{B. Comparison of DFT functionals}

Materials such as graphite, $h$-BN, and $\mathrm{MoS}_{2}$ have a layered structure, where the atoms within one layer form strong covalent bonds, but the interaction between the layers consists of weak, van der Waals, forces. Common GGA functionals, such as PBE [31], lack a description of van der Waals interactions, which results in a severe underestimation of the interlayer binding energy in graphite and $h$-BN, and an overestimation of the interlayer bonding distance [34,35]. Similar problems are encountered when graphene or $h$-BN are adsorbed on a metal substrate [25,27-30]. The LDA functional also lacks a description of van der Waals interactions, but it, somewhat fortuitously, gives reasonable binding energies and geometries for graphite, $h-\mathrm{BN}$, and for the adsorption of these materials on metals $[14,15,24,26]$. In general, however, the LDA functional tends to overestimate binding energies, which is regularly accompanied by an underestimation of the bonding distance.

Many of these problems are mitigated when using vdW-DFs $[46,47]$, which, for instance, describe the bonding in graphite very well [35]. The exchange-correlation energy in a vdW-DF takes the form

$$
E_{\mathrm{xc}}=E_{\mathrm{x}}+E_{\mathrm{c}}^{\mathrm{vdW}}+E_{\mathrm{c}}^{\mathrm{loc}},
$$

where $E_{\mathrm{x}}, E_{\mathrm{c}}^{\mathrm{loc}}$, and $E_{\mathrm{c}}^{\mathrm{vdW}}$ describe the exchange part, and the local and nonlocal electron-electron correlations, respectively. For $E_{\mathrm{c}}^{\mathrm{vdW}}$ we use the vdW kernel developed by Dion et al. [36] and for $E_{\mathrm{c}}^{\mathrm{loc}}$ the correlation part of the LDA functional. For the exchange part $E_{\mathrm{x}}$ we use the optB88 functional [38]. The opt88-vdW-DF has given good results for binding energies and geometries of graphite, $h$-BN, and the adsorption of these materials on metals $[14,35]$.

In the following, we test the GGA/PBE, LDA, and opt88vdW-DF functionals for the adsorption of $\mathrm{MoS}_{2}$ on metals. As test cases, we use the $4 d$ and $5 d$ metals $\mathrm{Ag}, \mathrm{Au}, \mathrm{Pd}$, and Pt. We place a $\mathrm{MoS}_{2}$ monolayer on top of the (111) surface of these metals, choosing a $\sqrt{3} \times \sqrt{3} R 30^{\circ}$ in-plane $\mathrm{MoS}_{2}$ unit cell on top of a $2 \times 2$ (111) surface cell. The in-plane $\mathrm{MoS}_{2}$ lattice parameters are kept at their optimized values for a free-standing layer, and the in-plane metal lattice parameter is adapted accordingly. The size of the adaption is maximal for $\mathrm{Au}$, where it results in a compression of the in-plane Au lattice by $4.2 \%$. The effects of this artificial strain are discussed in the next section.

(b)

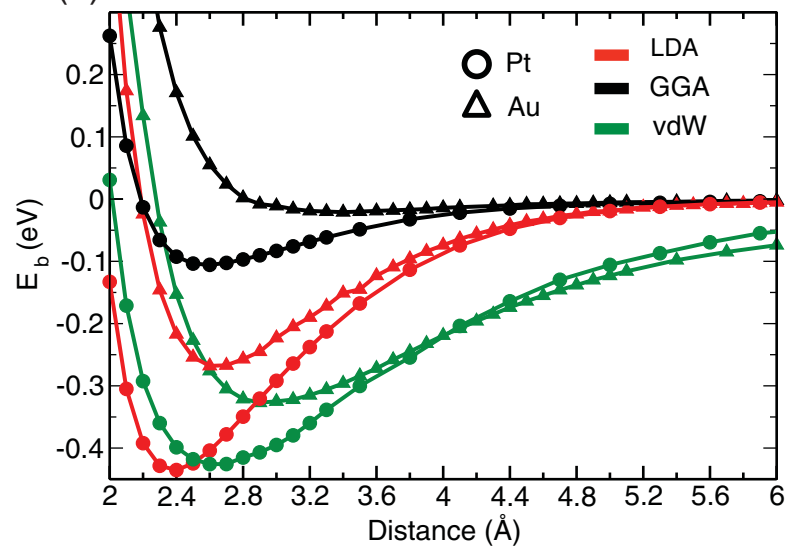

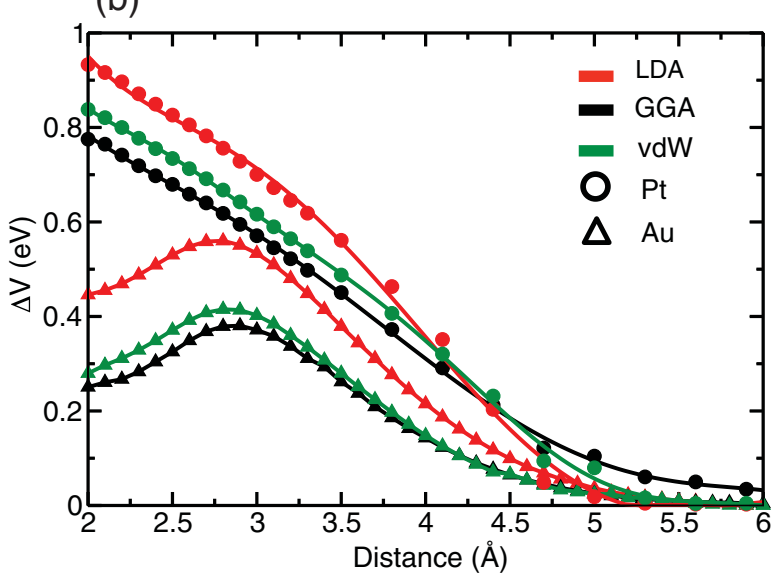

FIG. 2. (a) Binding energy curves $E_{b}(d)$ of $\mathrm{MoS}_{2}$ on $\mathrm{Au}$ and $\mathrm{Pt}(111)$, calculated with the GGA/PBE (black), opt $88 \mathrm{~b}$-vdw-DF (green), and LDA (red) functionals. (b) Interface potential steps $\Delta V(d)$ for $\mathrm{MoS}_{2}$ on $\mathrm{Au}$ and $\mathrm{Pt}(111)$, calculated with the three functionals. $d$ is the distance between the top metal and the bottom sulfur layers. 
TABLE I. Equilibrium bonding distance $d_{\mathrm{eq}}$, binding energy $E_{b}$, and interface potential step $\Delta V$, for $\mathrm{MoS}_{2}$ on metal (111) surfaces, calculated with different functionals.

\begin{tabular}{|c|c|c|c|c|c|c|c|c|c|c|c|c|}
\hline & \multicolumn{3}{|c|}{$\mathrm{Au}$} & \multicolumn{3}{|c|}{$\mathrm{Ag}$} & \multicolumn{3}{|c|}{$\mathrm{Pd}$} & \multicolumn{3}{|c|}{$\mathrm{Pt}$} \\
\hline & $d_{\mathrm{eq}}(\AA)$ & $\Delta V(\mathrm{eV})$ & $E_{b}(\mathrm{eV})$ & $d_{\mathrm{eq}}(\AA)$ & $\Delta V(\mathrm{eV})$ & $E_{b}(\mathrm{eV})$ & $d_{\text {eq }}(\AA)$ & $\Delta V(\mathrm{eV})$ & $E_{b}(\mathrm{eV})$ & $d_{\text {eq }}(\AA)$ & $\Delta V(\mathrm{eV})$ & $E_{b}(\mathrm{eV})$ \\
\hline LDA & 2.6 & 0.54 & -0.27 & 2.5 & 0.10 & -0.33 & 2.2 & 0.50 & -0.69 & 2.4 & 0.85 & -0.43 \\
\hline PBE & 3.3 & 0.38 & -0.02 & 2.8 & 0.10 & -0.08 & 2.3 & 0.34 & -0.25 & 2.6 & 0.66 & -0.11 \\
\hline vdW-DF & 2.9 & 0.41 & -0.33 & 2.8 & 0.11 & -0.35 & 2.4 & 0.30 & -0.54 & 2.6 & 0.71 & -0.43 \\
\hline
\end{tabular}

Figure 2(a) shows the binding curves of $\mathrm{MoS}_{2}$ on $\mathrm{Au}$ and $\mathrm{Pt}(111)$ for the three functionals. The binding energy is defined as the total energy per $\mathrm{MoS}_{2}$ formula unit of the metal/ $\mathrm{MoS}_{2}$ slab minus the total energies of the clean metal slab and the free-standing $\mathrm{MoS}_{2}$ layer, as a function of the distance $d$ between the top layer of metal atoms and the bottom layer of sulfur atoms. For $\mathrm{MoS}_{2}$ on $\mathrm{Au}(111)$, PBE gives virtually no bonding, and opt88-vdW-DF gives a sizable binding energy. The opt88-vdW-DF result suggests that $\mathrm{MoS}_{2}$ is physisorbed on $\mathrm{Au}(111)$, with van der Waals interactions playing the decisive role in the bonding. PBE does not capture this at all. LDA gives an equilibrium binding distance that is $0.3 \AA$ smaller, and an equilibrium binding energy that is $32 \%$ larger.

For $\mathrm{MoS}_{2}$ on $\mathrm{Pt}(111)$ all three functionals give equilibrium bonding distances that are shorter than for $\mathrm{MoS}_{2}$ on $\mathrm{Au}(111)$, and a bonding that is stronger, which suggests that $\mathrm{MoS}_{2}$ may be weakly chemisorbed on Pt(111). PBE and opt88-vdW-DF give a similar equilibrium distance, although PBE captures only $26 \%$ of the binding energy, indicating that van der Waals interactions still play an important role here. LDA gives a similar binding energy as opt88-vdW-DF, but an equilibrium binding distance that is $0.2 \AA$ smaller.

Table I shows the equilibrium binding distances and energies obtained with the three functionals for $\mathrm{MoS}_{2}$ on $\mathrm{Au}$, $\mathrm{Ag}, \mathrm{Pd}$, and $\mathrm{Pt}(111)$. Treating the results for opt88-vdW-DF as a benchmark, PBE is seen to severely underestimate binding energies, whereas LDA gives quite reasonable binding energies. LDA, however, gives binding distances that are up to $0.3 \AA$ shorter than those obtained with opt88-vdW-DF, in particular for cases where the bonding is weak, such as Au and Ag. In contrast, PBE gives binding distances that are similar to those obtained with opt88-vdW-DF, except for $\mathrm{Au}$, where PBE essentially fails to give any significant bonding.

Potential steps $\Delta V$ as a function of the distance $d$ between the top layer of metal atoms and the bottom layer of sulfur atoms, calculated according to Eq. (3), are shown in Fig. 2(b) for $\mathrm{Au}$ and Pt. The curves for the PBE and the opt88-vdW-DF functionals are within $0.05 \mathrm{eV}$ of one another in the range $d=2.5-3 \AA$, whereas LDA gives a potential step that is $0.10-$ $0.15 \mathrm{eV}$ higher. In view of the considerable differences in the binding curves for these three functionals, the differences in the potential steps are remarkably small. This is true for all metal substrates listed in Table I.

In Ref. [15] the main contribution to the potential step in the adsorption of $h$-BN on metal substrates was attributed to Pauli repulsion. This can be modeled by an electron density that is obtained by antisymmetrizing the product of the metal and the adsorbate wave functions. As long as these wave functions do not strongly depend on the functional, the electron density and the potential step are also relatively insensitive to the functional used. This is unlike the total energy, which for a given electron density is very dependent on the functional. For the potential step to be accurate it is, however, important to obtain the proper equilibrium binding distance $[14,15,24,26]$.

\section{Lattice mismatch}

The absolute values of the binding energies given in Table I are much smaller than what one expects to find for true chemical bonding. The differences between the values obtained with PBE and opt88-vdW-DF indicate that van der Waals interactions play a significant role in the bonding. With such a weak metal/adsorbate bonding it is unlikely that the metal substrate can enforce its lattice periodicity onto the $\mathrm{MoS}_{2}$ overlayer. Therefore, a metal/MoS $\mathrm{S}_{2}$ interface very likely becomes incommensurable if the metal/ $\mathrm{MoS}_{2}$ lattice mismatch is substantial. In electronic-structure calculations, one is forced to use commensurable structures to model incommensurable systems. Obviously, care must be taken to ensure that the artificial strain introduced this way does not alter the electronic structure in an unrealistic way.

Based upon previous experience, we expect that modifying the in-plane lattice constant of a close-packed metal surface by a few percent only affects its electronic properties mildly $[14,15,24,26]$. In contrast, changing the lattice parameter of $\mathrm{MoS}_{2}$ by just $1 \%$ already alters the band gap by $\sim 0.1 \mathrm{eV}$, and changes it from direct to indirect. A larger change in the lattice parameter has an even more dramatic effect. Applying a tensile strain of $\sim 5 \%$ to $\mathrm{MoS}_{2}$ reduces the band gap by $\sim 1 \mathrm{eV}$ [48-53].

As an example, the PBE optimized in-plane lattice parameters of $\mathrm{MoS}_{2}$ and $\mathrm{Au}(111)$ are 3.19 and $2.88 \AA$. Placing a $(\sqrt{3} \times \sqrt{3}) R 30^{\circ} \mathrm{MoS}_{2}$ cell on top of $2 \times 2 \mathrm{Au}(111)$ surface cell then leads to a lattice mismatch of $4.2 \%$. Figure $3(\mathrm{~b})$ shows the electronic band structure of $\mathrm{MoS}_{2} / \mathrm{Au}(111)$ where the in-plane $\mathrm{Au}(111)$ is compressed by $4.2 \%$ to match the lattice parameter of $\mathrm{MoS}_{2}$. As the interaction between $\mathrm{MoS}_{2}$ and the Au surface is relatively small, it is not surprising to see that the band structure of adsorbed $\mathrm{MoS}_{2}$ resembles that of free-standing $\mathrm{MoS}_{2}$, shown in Fig. 3(a). Note that in the $\sqrt{3} \times \sqrt{3} \mathrm{MoS}_{2}$ cell the bands are folded such that the direct band gap appears at the $\Gamma$ point. The work function of clean $\mathrm{Au}(111)$ is changed by only $0.08 \mathrm{eV}$ by the $4.2 \%$ compression of its lattice.

For comparison, Fig. 3(c) shows the band structure of $\mathrm{MoS}_{2} / \mathrm{Au}(111)$ when $\mathrm{MoS}_{2}$ is stretched by $4.2 \%$ to match the $\mathrm{Au}(111)$ lattice. Clearly, the band structure of $\mathrm{MoS}_{2}$ is 


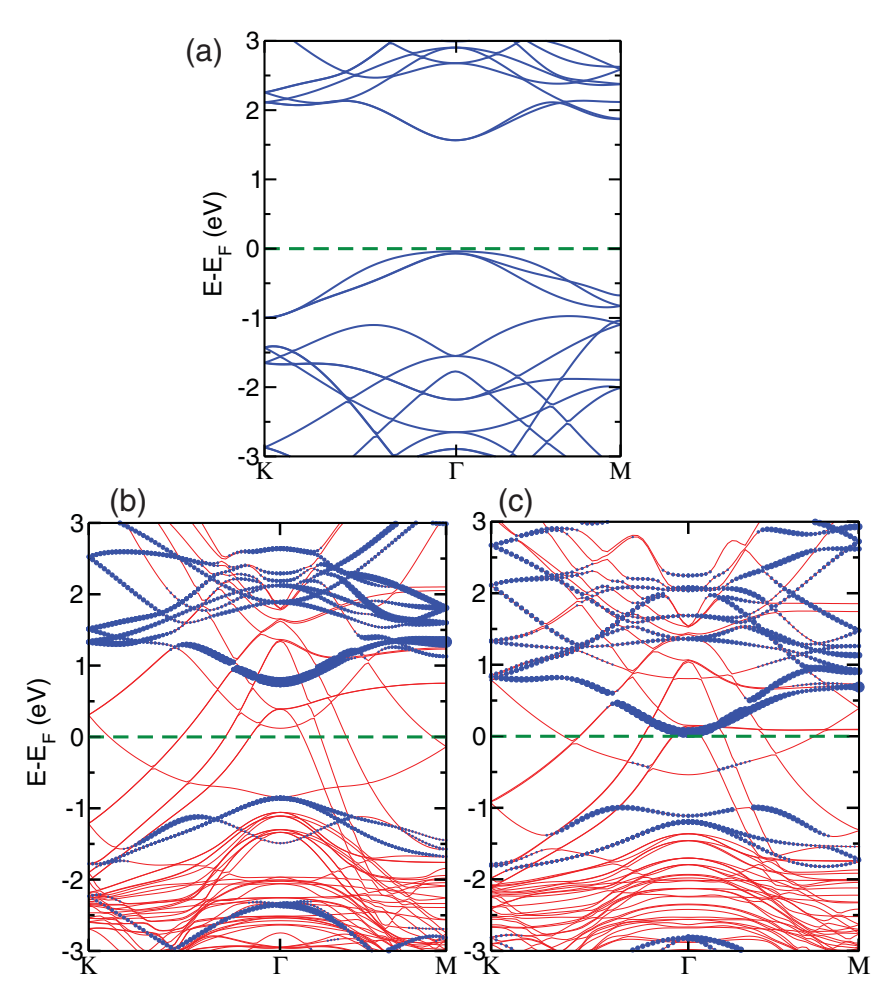

FIG. 3. (a) Band structure of a free-standing $\mathrm{MoS}_{2}$ monolayer in a $\sqrt{3} \times \sqrt{3}$ cell, where the direct band gap appears at $\Gamma$; (b) band structure of $\mathrm{MoS}_{2} / \mathrm{Au}(111)$ with the in-plane Au lattice compressed by $4.2 \%$ to match the $\mathrm{MoS}_{2}$ lattice; the blue color indicates the weight of a projection of the wave functions on the $\mathrm{MoS}_{2}$ sites; (c) as (b) but with the $\mathrm{MoS}_{2}$ lattice stretched by $4.2 \%$ to match the $\mathrm{Au}(111)$ lattice.

now changed significantly. It no longer shows a direct band gap at $\Gamma$, but an indirect band gap, and the size of the band gap is reduced to $\sim 1 \mathrm{eV}$, which is consistent with previous studies [48-53]. The Schottky barrier for electrons (the energy difference between the bottom of the conduction band and the Fermi level), which is a sizable $0.7 \mathrm{eV}$ in Fig. 3(b), is reduced to zero in Fig. 3(c) as in Ref. [19]. The latter is clearly unphysical: one would not expect a high-work-function metal such as Au to form a barrierless contact for electrons. Indeed, experimentally $\mathrm{Au}$ is found to form a substantial Schottky barrier with $\mathrm{MoS}_{2}[7,10,11,54,55]$.

In the following, we base the in-plane lattice constant of the $\mathrm{MoS}_{2} /$ metal slab on the optimized values of freestanding $\mathrm{MoS}_{2}$, which are 3.13, 3.18, and $3.19 \AA$ for the LDA, optb88-vdW-DF, and PBE functionals, respectively. Experimentally reported bulk $\mathrm{MoS}_{2}$ lattice constants are in the range 3.13-3.16 $\AA$ [56-58], suggesting that the LDA result may be more accurate and both PBE and the vdW functional are overestimating the lattice constant somewhat.

In making a commensurable structure, we adapt the metal to the $\mathrm{MoS}_{2}$ lattice. To minimize the artificial strain that is introduced by this adaptation, we construct in-plane supercells following the procedure of Ref. [59]. We denote a basis vector of a $\mathrm{MoS}_{2}$ supercell by $\vec{T}_{1}=n_{1} \vec{a}_{1}+n_{2} \vec{a}_{2}$, with $\left\{\vec{a}_{1}, \vec{a}_{2}\right\}$ the basis vectors of the primitive cell, and $n_{1}, n_{2}$ integers. Similarly, $\vec{T}_{1}^{\prime}=m_{1} \vec{b}_{1}+m_{2} \vec{b}_{2}$ is a basis vector of a metal surface supercell, with $\left\{\vec{b}_{1}, \vec{b}_{2}\right\}$ the basis vectors of the primitive

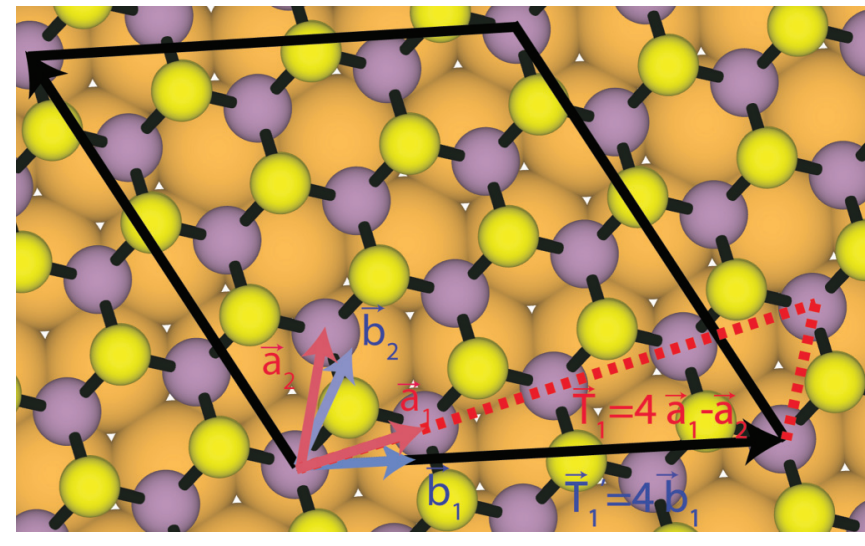

FIG. 4. Top view of $\mathrm{MoS}_{2} / \mathrm{Au}(111)$ interface indicating the supercell (black lines), the primitive basis vectors $\vec{a}_{1}, \vec{a}_{2}$ and $\vec{b}_{1}, \vec{b}_{2}$ of the $\mathrm{MoS}_{2}$ and $\mathrm{Au}(111)$ lattices, respectively, and the basis vectors $\vec{T}_{1}$ and $\vec{T}_{1}^{\prime}$ of the supercell.

cell, and $m_{1}, m_{2}$ integers. We search for a set of values for $n_{1}$, $n_{2}, m_{1}$, and $m_{2}$, such that the difference between the $\mathrm{MoS}_{2}$ and the metal supercell basis vectors is less than a margin $\delta$ :

$$
\frac{\left|\vec{T}_{1}\right|-\left|\vec{T}_{1}^{\prime}\right|}{\left|\vec{T}_{1}\right|} \leqslant \delta .
$$

We then rotate the $\mathrm{MoS}_{2}$ lattice by an angle $\alpha$ such that the directions of the $\vec{T}_{1}$ and $\vec{T}_{1}^{\prime}$ vectors coincide. Because of the symmetry of the lattice, the second basis vector of the supercell is easily obtained by a $120^{\circ}$ rotation $\vec{T}_{2}=$ $R\left(120^{\circ}\right) \vec{T}_{1}$. The commonly used surface science notation of this supercell is a $\sqrt{N} \times \sqrt{N} R \alpha \mathrm{MoS}_{2}$ lattice on top of a $\sqrt{M} \times \sqrt{M}$ metal lattice, where $N=n_{1}^{2}+n_{2}^{2}+n_{1} n_{2}$ and $M=m_{1}^{2}+m_{2}^{2}+m_{1} m_{2}$.

The parameter $\delta$ determines the mismatch between the $\mathrm{MoS}_{2}$ and the metal lattices, and the strain we apply to the metal lattice. In this study, we choose the smallest supercell for which $\delta<1 \%$. Figure 4 gives an example of a supercell for $\mathrm{MoS}_{2}$ on $\mathrm{Au}(111)$ that is constructed this way, and Table II lists the supercells and the lattice mismatch $\delta$ used in this study for the different metals.

TABLE II. In-plane supercell defined by the $\mathrm{MoS}_{2}$ lattice vector $R(\alpha) \vec{T}_{1}$, where $\vec{T}_{1}=n_{1} \vec{a}_{1}+n_{2} \vec{a}_{2}$ and the metal lattice vector $\vec{T}_{1}^{\prime}=$ $m_{1} \vec{b}_{1}+m_{2} \vec{b}_{2} . \delta$ gives the mismatch between the $\mathrm{MoS}_{2}$ and metal lattices [Eq. (7)] (PBE values).

\begin{tabular}{lcccl}
\hline \hline & $n_{1}, n_{2}$ & $m_{1}, m_{2}$ & $\alpha$ & $\delta(\%)$ \\
\hline $\mathrm{Mg}$ & 1,0 & 1,0 & $0^{\circ}$ & 0.6 \\
$\mathrm{Al}$ & $4,-1$ & 4,0 & $13.9^{\circ}$ & 0.5 \\
$\mathrm{Ag}$ & $4,-1$ & 4,0 & $13.9^{\circ}$ & 0.15 \\
$\mathrm{Ti}$ & $5,-2$ & 4,0 & $23.4^{\circ}$ & 0.7 \\
$\mathrm{Cu}$ & 4,0 & 5,0 & $0^{\circ}$ & 0.3 \\
$\mathrm{Au}$ & $4,-1$ & 4,0 & $13.9^{\circ}$ & 0.15 \\
$\mathrm{Pd}$ & 1,1 & 2,0 & $30^{\circ}$ & 0.3 \\
$\mathrm{Pt}$ & 1,1 & 2,0 & $30^{\circ}$ & 0.3 \\
$\mathrm{Co}$ & $5,-4$ & $4,-3$ & $3^{\circ}$ & 0.01 \\
$\mathrm{Ni}$ & $5,-4$ & $4,-3$ & $3^{\circ}$ & 0.8 \\
\hline \hline
\end{tabular}




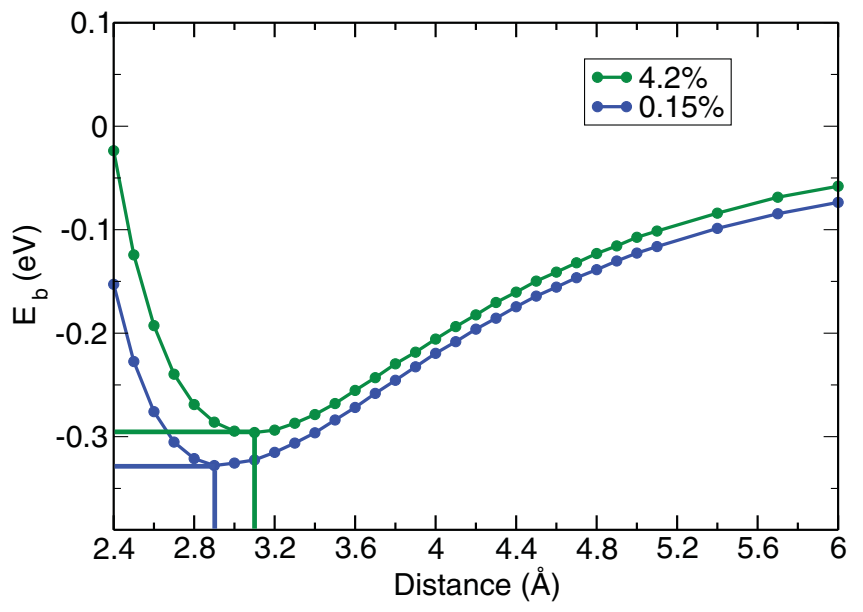

FIG. 5. Binding energy curves $E_{b}(d)(\mathrm{eV})$ of $\mathrm{MoS}_{2}$ on $\mathrm{Au}(111)$, calculated with opt8b-vdW-DF functional for a mismatch of $0.15 \%$ (blue), and $4.2 \%$ (green) between the $\mathrm{MoS}_{2}$ and the $\mathrm{Au}(111)$ lattices.

In the calculations discussed in Secs. II B and II C, we have used a $\sqrt{3} \times \sqrt{3} R 30^{\circ} \mathrm{MoS}_{2}$ cell on top of a $2 \times 2$ $\mathrm{Au}(111)$ cell, which leads to a lattice mismatch of $4.2 \%$. A $\sqrt{13} \times \sqrt{13} R 13.9^{\circ}$ on top of a $4 \times 4$ Au supercell (see Table II and Fig. 4) reduces the lattice mismatch to $0.15 \%$. Figure 5 shows that the binding energy curves for the two structures are quite similar. The equilibrium binding energy is increased by $0.03 \mathrm{eV}$ upon compressing the Au lattice by $4.2 \%$, and the equilibrium binding distance is decreased by $0.2 \AA$. Typically, the interface potential step is affected by the compression on a scale of $0.1 \mathrm{eV}$, as is shown in Table III. However, sometimes the effect is larger, as for Ag. In conclusion, compressing the metal lattice does not generally have the same dramatic effect as stretching the $\mathrm{MoS}_{2}$ lattice has, but large lattice mismatches should be avoided.

\section{RESULTS}

\section{A. Metal/MoS $\mathbf{M}_{2}$ interaction}

Calculated equilibrium binding energies and bonding distances for the $\mathrm{MoS}_{2} /$ metal structures of Table II are listed in Table IV. The binding energies obtained with opt88$\mathrm{vdW}-\mathrm{DF}$ are in the range -0.3 to $-0.6 \mathrm{eV}$. These numbers seem somewhat too low in order to classify the bonding as physisorption, yet too high to call it chemisorption. Van der Waals interactions play an important role in the bonding, which becomes especially clear when comparing to the results obtained by PBE. The PBE functional lacks van der Waals

TABLE III. Equilibrium bonding distance $d_{\text {eq }}$, binding energy $E_{b}$, and interface potential step $\Delta V$ for $\mathrm{MoS}_{2}$ on metal (111) surfaces, calculated with supercell lattices with a different mismatch $\delta$.

\begin{tabular}{lccccccc}
\hline \hline & \multicolumn{4}{c}{$\mathrm{Au}$} & & \multicolumn{3}{c}{$\mathrm{Ag}$} \\
\cline { 2 - 4 } \cline { 6 - 8 }$\delta(\%)$ & $d_{\text {eq }}(\AA)$ & $\Delta V(\mathrm{eV})$ & $E_{b}(\mathrm{eV})$ & & $d_{\text {eq }}(\AA)$ & $\Delta V(\mathrm{eV})$ & $E_{b}$ \\
\hline 0.15 & 2.9 & 0.41 & -0.33 & & 2.8 & 0.11 & -0.35 \\
4.2 & 3.1 & 0.51 & -0.30 & & 2.9 & 0.47 & -0.32 \\
\hline \hline
\end{tabular}

TABLE IV. Equilibrium binding energy $E_{b}$ and bonding distance $d_{\mathrm{eq}}$ for $\mathrm{MoS}_{2}$ on metal (111) and (0001) surfaces in the interface structures of Table II, calculated with the optb88b-vdW-DF and the PBE functionals.

\begin{tabular}{lcccc}
\hline \hline & $\begin{array}{c}E_{\mathrm{b}(\mathrm{vdW})} \\
(\mathrm{eV})\end{array}$ & $\begin{array}{c}d_{\mathrm{eq}(\mathrm{vdW})} \\
(\AA)\end{array}$ & $\begin{array}{c}E_{\mathrm{b}(\mathrm{PBE})} \\
(\mathrm{eV})\end{array}$ & $\begin{array}{c}d_{\mathrm{eq}(\mathrm{PBE})}(\AA) \\
(\mathrm{A})\end{array}$ \\
\hline $\mathrm{Mg}$ & -0.55 & 2.3 & -0.20 & 2.2 \\
$\mathrm{Al}$ & -0.30 & 2.8 & -0.30 & 2.8 \\
$\mathrm{Ag}$ & -0.35 & 2.8 & -0.08 & 2.9 \\
$\mathrm{Ti}$ & -0.51 & 2.3 & -0.67 & 2.3 \\
$\mathrm{Cu}$ & -0.40 & 2.5 & -0.16 & 2.4 \\
$\mathrm{Au}$ & -0.33 & 2.9 & -0.02 & 3.3 \\
$\mathrm{Pd}$ & -0.54 & 2.4 & -0.25 & 2.3 \\
$\mathrm{Ni}$ & -0.51 & 2.2 & -0.25 & 2.2 \\
$\mathrm{Co}$ & -0.57 & 2.2 & -0.29 & 2.2 \\
$\mathrm{Pt}$ & -0.43 & 2.6 & -0.11 & 2.6 \\
\hline \hline
\end{tabular}

interactions, and it typically captures only approximately half the $\mathrm{MoS}_{2} /$ metal binding energy or less.

A noticeable exception is $\mathrm{MoS}_{2} / \mathrm{Ti}(0001)$, where PBE gives approximately double the opt88-vdW-DF binding energy. It suggests that $\mathrm{MoS}_{2}$ is chemisorbed on $\mathrm{Ti}(0001)$, which is described better by PBE. This case will be discussed in more detail in Sec. III C. In contrast, the PBE functional essentially fails to give bonding for the adsorption of $\mathrm{MoS}_{2}$ on $\mathrm{Au}(111)$, and all bonding comes from van der Waals interactions, so we may classify this case as physisorption. For the other metals, it is difficult to make a distinction between physisorption and chemisorption on the basis of the binding energy alone.

In general terms, physisorption is accompanied by a weak perturbation of the electronic structure of the adsorbed layer, whereas chemisorption results in a sizable perturbation of that electronic structure. For graphene and $h$-BN adsorbed on metal surfaces it is possible to correlate that perturbation with the equilibrium bonding distances $d_{\text {eq. }}$. Those distances can be divided into two groups separated by a critical binding distance $d_{\mathrm{c}}$. For $d_{\mathrm{eq}}>d_{\mathrm{c}}$, the bonding is physisorption, and for $d_{\text {eq }}<d_{\mathrm{c}}$, the bonding is chemisorption. For graphene and $h$-BN this distinction is successful because there are hardly any cases where $d_{\mathrm{eq}} \approx d_{\mathrm{c}} \approx 2.8 \AA$ as is illustrated in Fig. 6. Clearly, bonding distances and energies are correlated; a shorter distance generally gives a lower energy.

Plotting the binding energies and distances for $\mathrm{MoS}_{2} /$ metal interfaces in Fig. 6, one observes that the distinction between physisorption and chemisorption is much less clear for this case. The binding of $\mathrm{MoS}_{2}$ to a metal substrate is stronger than that of graphene or $h$-BN, reflecting the fact that van der Waals interactions increase with the atomic number. Maybe somewhat surprisingly the bonding distance of $\mathrm{MoS}_{2}$ to a metal substrate is generally shorter than that of graphene or $h$-BN. Graphene and $h$-BN have $\pi$ orbitals that stick out below their respective planes, which give rise to a substantial Pauli repulsion at distances to the metal plane of $\lesssim 3 \AA$ [15]. Apparently, the wave functions of $\mathrm{MoS}_{2}$ do not stick out that far below the plane of the bottom sulfur layer.

The bonding distances for $\mathrm{MoS}_{2} /$ metal interfaces cannot easily be divided into two groups, as is the case for graphene and $h$-BN/metal interfaces. Instead, there is a more gradual 


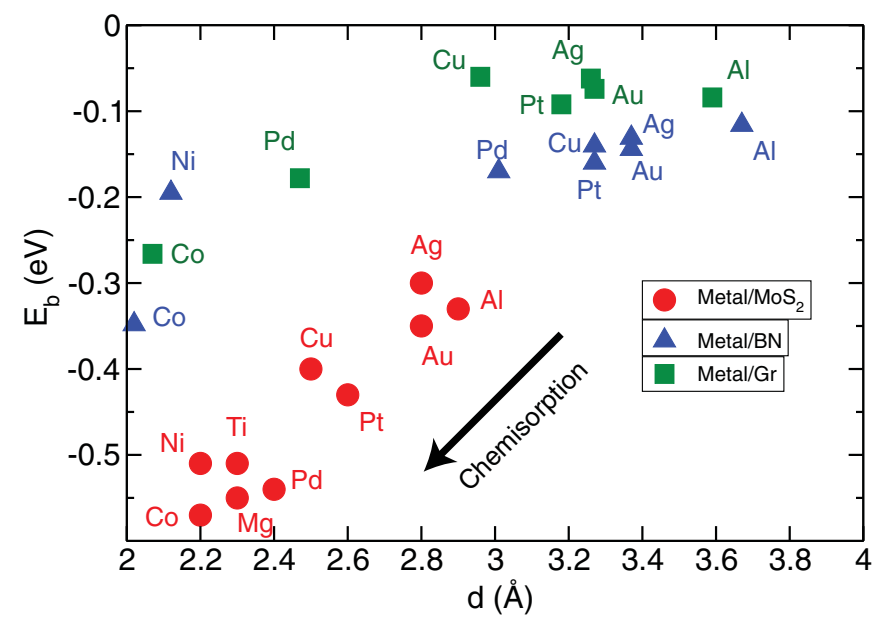

FIG. 6. Binding energy $E_{b}(\mathrm{eV})$ per $\mathrm{MoS}_{2}$ versus equilibrium bonding distance $d_{\mathrm{eq}}(\AA)$ for $\mathrm{MoS}_{2}$ adsorbed on metal(111) and (0001) substrates (red circles), as calculated with the optb88-vdW-DF functional. For comparison, results for $h$-BN (blue triangles) [14] and graphene (green squares) [60] are also shown.

scale. The bonding distances of $\mathrm{MoS}_{2}$ on $\mathrm{Al}, \mathrm{Au}$, and $\mathrm{Ag}$ are on the physisorption side of Fig. 6, whereas on $\mathrm{Co}, \mathrm{Ni}$, $\mathrm{Mg}$, and $\mathrm{Ti}$, they are more on the chemisorption side, with $\mathrm{Pt}$, $\mathrm{Cu}, \mathrm{Pd}$ as intermediate cases. However, a clear dividing line like for graphene and $h$-BN can not be drawn. Indeed, if one considers the $\mathrm{MoS}_{2} /$ metal interface for two similar metals that give rise to a fairly large difference in bonding distance and binding energy, $\mathrm{Ag}$ and $\mathrm{Pd}$, one does not observe a qualitative difference in the the electronic structure of the $\mathrm{MoS}_{2}$ adsorbate (see Fig. 7). In both cases, the $\mathrm{MoS}_{2}$ bands are perturbed by the metal- $\mathrm{MoS}_{2}$ interaction, but the signature of the $\mathrm{MoS}_{2}$ bands can still be recognized. In particular, it still seems to be possible to identify the top of the $\mathrm{MoS}_{2}$ valence band and the bottom of the conduction band. Nevertheless, the $\mathrm{MoS}_{2}$ states do hybridize with those of the metal substrate, as we will discuss in the next section.

Another way of characterizing the bonding is to analyze the geometry of the $\mathrm{MoS}_{2} /$ metal interface. Chemisorption involves
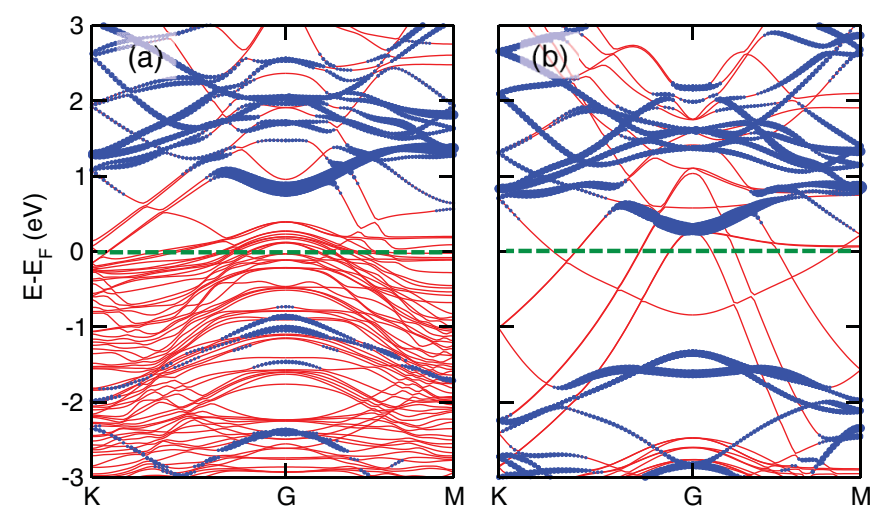

FIG. 7. (a) Band structure of $\mathrm{MoS}_{2} / \mathrm{Pd}(111)$; the blue color indicates the weight of a projection of the wave functions on the $\mathrm{MoS}_{2}$ sites; (b) idem for $\mathrm{MoS}_{2} / \mathrm{Ag}(111)$; for comparison both band structures are shown in a $\sqrt{3} \times \sqrt{3}$ surface cell.
TABLE V. The average displacements $\bar{\Delta}_{\mathrm{S}}$ and $\bar{\Delta}_{\mathrm{M}}$ of the bottom layer of sulfur atoms and of the top layer of metal atoms, and the corresponding maximum displacements $\Delta_{\mathrm{S}}^{\max }$ and $\Delta_{\mathrm{M}}^{\max }$, after the adsorption.

\begin{tabular}{lcccc}
\hline \hline & $\bar{\Delta}_{\mathrm{S}}(\AA)$ & $\Delta_{\mathrm{S}}^{\max }(\AA)$ & $\bar{\Delta}_{\mathrm{M}}(\AA)$ & $\Delta_{\mathrm{M}}^{\max }(\AA)$ \\
\hline $\mathrm{Ti}$ & 0.101 & 0.152 & 0.176 & 0.483 \\
$\mathrm{Co}$ & 0.010 & 0.021 & 0.068 & 0.102 \\
$\mathrm{Pt}$ & 0.006 & 0.009 & 0.049 & 0.098 \\
$\mathrm{Ag}$ & 0.002 & 0.005 & 0.030 & 0.058 \\
$\mathrm{Au}$ & 0.001 & 0.004 & 0.019 & 0.044 \\
$\mathrm{Pd}$ & 0.002 & 0.003 & 0.026 & 0.051 \\
$\mathrm{Ni}$ & 0.017 & 0.034 & 0.089 & 0.150 \\
$\mathrm{Al}$ & 0.001 & 0.047 & 0.046 & 0.105 \\
$\mathrm{Mg}$ & 0.022 & 0.022 & 0.091 & 0.091 \\
$\mathrm{Cu}$ & 0.033 & 0.052 & 0.070 & 0.124 \\
\hline \hline
\end{tabular}

the formation of chemical bonds between the adsorbate and the metal, which frequently also leads to a deformation of the adsorbate's structure. We can define a displacement $\Delta_{i}=\left|\mathbf{R}_{i}-\mathbf{R}_{0, i}\right|$ between the position $\mathbf{R}_{i}$ of an atom $i$ in the optimized $\mathrm{MoS}_{2} /$ metal structure and its position $\mathbf{R}_{0, i}$ in the free-standing $\mathrm{MoS}_{2}$ or in the clean metal substrate. The displacements are obtained in a two-step procedure. First, the $\mathrm{MoS}_{2} /$ metal structure is optimized while freezing the $\mathrm{MoS}_{2}$ layer and the metal substrate in their free-standing geometries. Once the equilibrium distance $d_{\text {eq }}$ is obtained, as in Figs. 2 and 5, all atomic positions are relaxed, and this last step defines the displacements. Table $\mathrm{V}$ gives $\bar{\Delta}_{\mathrm{S}}$ and $\bar{\Delta}_{\mathrm{M}}$, which are the average displacements of the bottom layer of sulfur atoms, and of the top layer of metal atoms, respectively, for some representative metal substrates. In addition, this table gives the maximum displacements $\Delta_{\mathrm{S}}^{\max }$ and $\Delta_{\mathrm{M}}^{\max }$.

The displacements are quite large for the $\mathrm{MoS}_{2} / \mathrm{Ti}(0001)$ structure, indicating that there is a significant distortion of the geometries of both the $\mathrm{MoS}_{2}$ adsorbate and the Ti surface, which strongly suggests that $\mathrm{MoS}_{2}$ is chemisorbed on Ti. At the opposite end of the scale, we find $\mathrm{MoS}_{2} / \mathrm{Au}(111)$, where the atomic displacements are small, indicating that here we are in the physisorption regime. The behavior of the other metal substrates is in-between these two extreme cases but more to the physisorption side. The $3 d$ transition metals $\mathrm{Co}, \mathrm{Ni}, \mathrm{Cu}$ and the low-work-function simple metal $\mathrm{Mg}$ show somewhat larger distortions than the $4 d$ and $5 d$ metals $\mathrm{Pd}, \mathrm{Ag}, \mathrm{Pt}$ and the simple metal Al.

\section{B. Interface potential step and Schottky barrier}

Table VI gives the interface potential steps $\Delta V$ created by the adsorption of $\mathrm{MoS}_{2}$ on a metal substrate. This potential step strongly influences the Schottky barrier at metal/ $\mathrm{MoS}_{2}$ contacts [see Eq. (5)], and as such it plays an important role in the physics of $\mathrm{MoS}_{2}$ semiconductor devices. The potential steps can be divided into two groups, i.e., positive $\Delta V$ for metals with a high work function, and negative $\Delta V$ for lowwork-function metals. A positive $\Delta V$ means that adsorption of $\mathrm{MoS}_{2}$ effectively lowers the work function of the substrate. The $\mathrm{MoS}_{2}$ layer has no intrinsic dipole moment perpendicular to the layer that could create such a potential step. So, the work 
function lowering is a purely electronic effect that results from the displacement of surface electron density into the metal by physisorption of the adsorbate.

This effect is known as the pushback effect or the pillow effect, which is a general phenomenon observed in the physisorption of closed-shell atoms, molecules, and layers on metal substrates. In Ref. [15], we have developed a quantitative model for this effect, based upon an antisymmetrization of the product of the metal and adsorbate wave functions. When an adsorbate approaches a metal surface, the wave functions of the two systems overlap. Pauli exchange repulsion between these states leads to a spatial redistribution of the electron density, in particular to a decrease of the density in the overlap region. Since the metal wave functions are usually more extended and more easily deformable than those of the adsorbate, the net result of this redistribution is that electrons are pushed back into the metal, which effectively lowers the work function.

In the adsorption of graphene and $h$-BN on high-workfunction metals, we found potentials steps of up to $1-2 \mathrm{eV}$. The potential steps for $\mathrm{MoS}_{2}$ adsorbed on the same metals are generally smaller, and more typically around $0.3-0.4 \mathrm{eV}$. The wave functions of first-row elements $(\mathrm{B}, \mathrm{C}, \mathrm{N})$ are compact and not easily deformable, as compared to the wave functions of the metal substrate. The effect of Pauli repulsion in the metal/adsorbate overlap region is then very asymmetric. It is foremost the metal electron density that is deformed, i.e., pushed back, which gives a large-work-function lowering. If the adsorbate contains heavier elements, such as $\mathrm{MoS}_{2}$, the effect of Pauli repulsion is more symmetric, i.e., both the metal and the adsorbate electrons are pushed out of the overlap region in a more symmetric way. This gives a smaller effect on the work function. Note that if the effect of Pauli repulsion would be completely symmetric, the work function would be unchanged.

Low-work-function metals experience an increase of the work function upon adsorption of $\mathrm{MoS}_{2}$, i.e., a negative $\Delta V$, which indicates a net transfer of electrons from the metal to the $\mathrm{MoS}_{2}$ adsorbate. As $\mathrm{MoS}_{2}$ is a semiconductor, it can only receive electrons in its conduction band. Therefore, for lowwork-function metal substrates one expects the Fermi level to be in the conduction band of $\mathrm{MoS}_{2}$. Analysis of the electronic structure of the $\mathrm{MoS}_{2} /$ metal slab, however, shows that this is not the case. The interaction between $\mathrm{MoS}_{2}$ and the metal at the interface leads to interface states with energies in the $\mathrm{MoS}_{2}$ band gap. That seems obvious if $\mathrm{MoS}_{2}$ is chemisorbed onto the substrate, as in the case of $\mathrm{MoS}_{2} / \mathrm{Ti}(0001)$, which we will discuss in the next section.

Somewhat surprisingly, a significant density of interface states also forms if the interaction between $\mathrm{MoS}_{2}$ and the metal substrate is relatively weak. For example, as discussed in the previous section, the interaction between $\mathrm{MoS}_{2}$ and $\mathrm{Al}(111)$ can be classified as physisorption (see Fig. 6). Nevertheless, states with energies inside the $\mathrm{MoS}_{2}$ band gap are formed at the $\mathrm{MoS}_{2} / \mathrm{Al}(111)$ interface, as is immediately obvious when comparing the density of states of the interface with that of free-standing $\mathrm{MoS}_{2}$ (see Fig. 8). The density of these interface states is not extremely high, yet sufficiently high to pin the Fermi level below the $\mathrm{MoS}_{2}$ conduction band, as demonstrated by Fig. 8. The density of interface states increases with increasing $\mathrm{MoS}_{2}$ /metal interaction, but even for

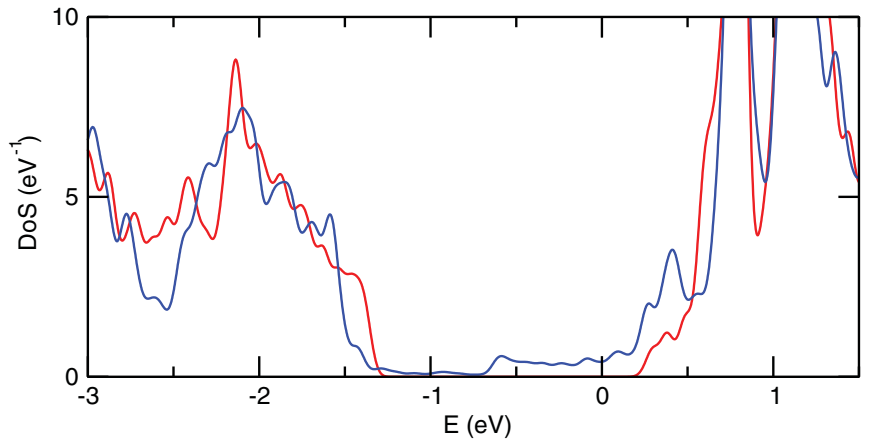

FIG. 8. (Blue) The total density of states of the $\mathrm{MoS}_{2} / \mathrm{Al}(111)$ slab; (red) the sum of the densities of states of free-standing $\mathrm{MoS}_{2}$ and of the $\mathrm{Al}(111)$ slab. The densities of states are aligned by aligning the Mo $4 s$ core levels and the $\mathrm{Al} 2 p$ core levels.

physisorption it seems sufficiently high to prevent the Fermi level from reaching the $\mathrm{MoS}_{2}$ conduction band.

One can prove that these interface states are indeed responsible for pinning the Fermi level by artificially enlarging the distance between the $\mathrm{MoS}_{2}$ layer and the metal surface. This breaks the direct $\mathrm{MoS}_{2} /$ metal contact that is responsible for the formation of interface states. In the absence of interface states, the Fermi level is at the bottom of the $\mathrm{MoS}_{2}$ conduction band [see Fig. 9(d)], which is what one would expect if the work function of the metal $W_{\mathrm{M}}$ is smaller than the electron affinity of $\mathrm{MoS}_{2} \chi_{\mathrm{MoS}_{2}}$ [see Eq. (5)]. A transfer of electrons between the metal and the $\mathrm{MoS}_{2}$ overlayer then yields a charge
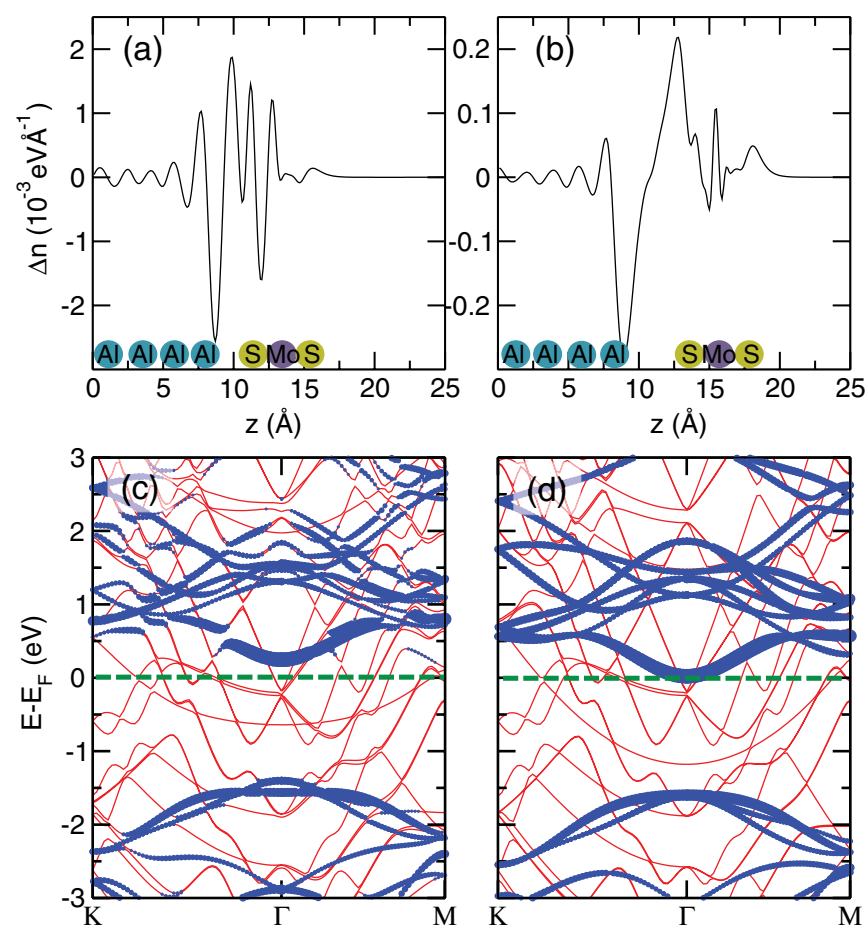

FIG. 9. (a) The electron density difference $\Delta n(z)$ of the $\mathrm{MoS}_{2} / \mathrm{Al}(111)$ interface at the equilibrium distance $d_{\mathrm{eq}}=3.2 \AA$, and (b) at a distance $d=6 \AA$; (c), (d) the corresponding band structures; the blue color indicates the weight of a projection of the wave functions on the $\mathrm{MoS}_{2}$ sites. 
distribution that can be associated with a simple interface dipole [see Fig. 9(b)]. In contrast, if interface states are formed, the band structure of adsorbed $\mathrm{MoS}_{2}$ is perturbed [see Fig. 9(c)] and the pattern of the charge distribution at the interface is much more complicated, as shown in Fig. 9(a). In that case, the interface states pin the Fermi level below the bottom of the $\mathrm{MoS}_{2}$ conduction band (see Fig. 8).

Schottky barrier heights (SBHs) for electrons, calculated according to Eq. (5), are also listed in Table VI. The functional causes some uncertainty, as the work functions of the clean metal surfaces obtained with the opt88-vdW-DF functional tend to be somewhat higher than those obtained with the PBE functional. LDA in general gives even higher work functions, so opt88-vdW-DF gives work functions that are in-between those of PBE and LDA [14,26]. Note that the interface potential steps $\Delta V$ do not depend strongly on the functional. As the opt88-vdW-DF functional also gives a larger electron affinity for $\mathrm{MoS}_{2}$, the Schottky barrier $\Phi_{\mathrm{n}}$ according to Eq. (5) also does not depend strongly on the functional.

Nevertheless, there is an uncertainty in the calculated SBHs coming from the $\mathrm{MoS}_{2}$ electron affinity. One may argue that the electron affinity calculated with either of the functionals is too high, as DFT band gaps are too small, which would give SBHs that are too small. The band gaps of a $\mathrm{MoS}_{2}$ monolayer calculated with the PBE and opt88-vdW-DF functionals are 1.63 and $1.67 \mathrm{eV}$, respectively, which can be compared to the experimental optical band gap of $1.86 \mathrm{eV}$ [4,61]. Such a comparison is not strictly fair, as the difference should reflect the exciton binding energy. Exciton binding energies between negligible [4] and $0.5 \mathrm{eV}$ [62] have been reported. Note, however, that the exciton binding energy strongly depends on screening by the environment $[62,63]$. For $\mathrm{MoS}_{2}$ adsorbed on metals one expects the screening to be large, and consequently the exciton binding energy to be relatively small.

We see that the SBH decreases with decreasing metal work function but does not go to zero. Instead, it goes through a minimum for $\mathrm{Cu}$ and $\mathrm{Ag}$, and then increases again for low-work-function metals like $\mathrm{Al}$ and $\mathrm{Mg}$. As discussed above, this phenomenon is caused by interface states. The only way to get rid of such states is to break the direct interaction between $\mathrm{MoS}_{2}$ and the metal substrate. Reference [13] discusses a practical way of doing this by inserting an atomic layer between the metal surface and the $\mathrm{MoS}_{2}$ layer. If this intermediate layer is purely van der Waals bonded to $\mathrm{MoS}_{2}$, no gap states are formed at its interface with $\mathrm{MoS}_{2}$. In addition, the intermediate layer should be transparent to electrons, such that the interface resistance is not dramatically increased. A monolayer of $h$-BN or graphene satisfies these criteria $[13,64,65]$.

\section{C. $\mathrm{MoS}_{2} / \mathrm{Ti}(0001)$}

As discussed in Sec. III A, $\mathrm{MoS}_{2}$ is chemisorbed on Ti(0001). The binding energy and the equilibrium bonding distance of $\mathrm{MoS}_{2}$ on $\mathrm{Ti}(0001)$ do not seem to be qualitatively different from those for $\mathrm{MoS}_{2}$ on metal substrates such as Co(0001) or Ni(111) (see Table IV and Fig. 6). However, the structural deformation of the $\mathrm{MoS}_{2}$ layer adsorbed on $\mathrm{Ti}(0001)$ is much larger than that of $\mathrm{MoS}_{2}$ on other metals (see Table V). This deformation is illustrated in Fig. 10. Atoms of the bottom
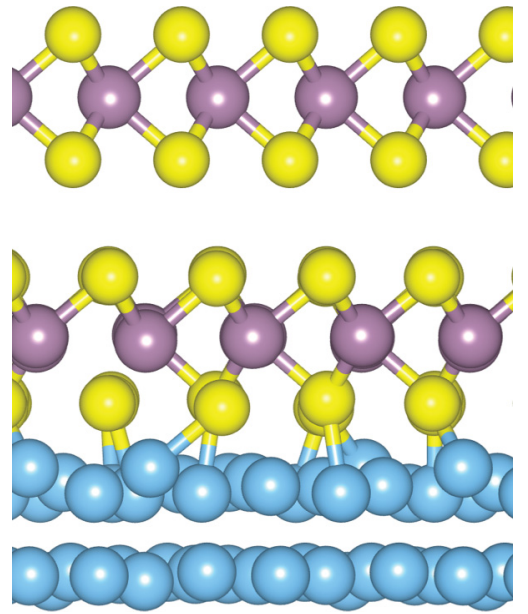

FIG. 10. Side view of two layers of $\mathrm{MoS}_{2}$ adsorbed on Ti(0001).

sulfur layer make a bond with $\mathrm{Ti}$ atoms of the top layer of the substrate, where several of these metal atoms are pulled up from the substrate. The $\mathrm{MoS}_{2}$ and the $\mathrm{Ti}(0001)$ lattices do not match very well; one needs a $\sqrt{19} \times \sqrt{19} R 23.4^{\circ}$ $\mathrm{MoS}_{2}$ supercell on top of a $4 \times 4 \mathrm{Ti}(0001)$ supercell to get a mismatch below $1 \%$ (see Table II). The result therefore is a $\mathrm{MoS}_{2} / \mathrm{Ti}(0001)$ interface that contains a substantial local strain, which explains why the binding energy is not very large, despite the bonding being chemisorption.

The potential step $\Delta V$ at the $\mathrm{MoS}_{2} / \mathrm{Ti}(0001)$ interface is negative, in contrast to the potential step at the $\mathrm{MoS}_{2} / \mathrm{Ag}(111)$ interface for instance, which is positive, despite the fact that the work functions of Ti and Ag are very similar (see Table VI). We argued that physisorption should lead to a positive potential step because of the Pauli repulsion effect, and indeed $\mathrm{MoS}_{2}$ is physisorbed on $\mathrm{Ag}(111)$. Chemisorption, as in the case of $\mathrm{MoS}_{2}$ on $\mathrm{Ti}(0001)$, leads to a more drastic reorganization of the charge distribution at the interface because of the formation of new chemical bonds. Upon the formation of these bonds, there is apparently a net displacement of electronic density towards the sulfur atoms, which is not unreasonable as sulfur

TABLE VI. Metal work function $W_{\mathrm{M}}$, interface potential step $\Delta V$, and Schottky barrier height $\Phi_{\mathrm{n}}$ calculated with the PBE and opt88-vdW-DF functionals, with calculated $\mathrm{MoS}_{2}$ electron affinities of $\chi=4.30$ and $4.57 \mathrm{eV}$, respectively.

\begin{tabular}{|c|c|c|c|c|c|c|}
\hline & \multicolumn{2}{|c|}{ PBE } & \multicolumn{4}{|c|}{ vdW-DF } \\
\hline & $W_{\mathrm{M}}(\mathrm{eV})$ & $\Delta V(\mathrm{eV})$ & $\Phi_{\mathrm{n}}(\mathrm{eV})$ & $W_{\mathrm{M}}(\mathrm{eV})$ & $\Delta V(\mathrm{eV})$ & $\Phi_{\mathrm{n}}(\mathrm{eV})$ \\
\hline $\mathrm{Mg}$ & 3.78 & -0.77 & 0.25 & 3.96 & -0.74 & 0.13 \\
\hline $\mathrm{Al}$ & 4.00 & -0.54 & 0.24 & 4.20 & -0.56 & 0.19 \\
\hline $\mathrm{Ag}$ & 4.47 & 0.10 & 0.07 & 4.82 & 0.11 & 0.14 \\
\hline $\mathrm{Ti}$ & 4.52 & -0.28 & $0.53^{\mathrm{a}}$ & 4.80 & -0.27 & $0.54^{\mathrm{a}}$ \\
\hline $\mathrm{Cu}$ & 4.70 & 0.35 & 0.05 & 5.10 & 0.39 & 0.14 \\
\hline $\mathrm{Au}$ & 5.30 & 0.32 & 0.68 & 5.58 & 0.41 & 0.60 \\
\hline $\mathrm{Pd}$ & 5.35 & 0.35 & 0.70 & 5.48 & 0.30 & 0.61 \\
\hline $\mathrm{Pt}$ & 5.75 & 0.64 & 0.81 & 5.96 & 0.68 & 0.71 \\
\hline Co & 5.13 & 0.29 & 0.54 & 5.42 & 0.34 & 0.51 \\
\hline $\mathrm{Ni}$ & 5.17 & 0.28 & 0.59 & 5.40 & 0.37 & 0.46 \\
\hline
\end{tabular}

${ }^{\mathrm{a}}$ See Sec. III C. 


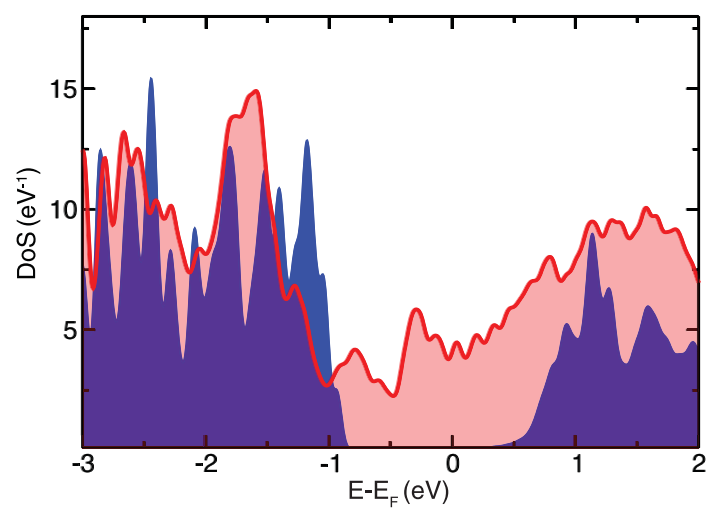

FIG. 11. The red and the blue shaded areas indicate the DOS projected on the first $\mathrm{MoS}_{2}$ layer of $\mathrm{MoS}_{2}$ adsorbed on Ti(0001) and on the second $\mathrm{MoS}_{2}$ layer, respectively.

is more electronegative than Ti. This displacement results in an increase of the work function, i.e., a negative $\Delta V$.

One expects that chemisorption also leads to a strong perturbation of the electronic structure of the adsorbate. Figure 11 shows the density of states (DOS) of a $\mathrm{MoS}_{2}$ bilayer projected on the individual $\mathrm{MoS}_{2}$ layers. The DOS of the first (chemisorbed) layer is indeed strongly perturbed as compared to the DOS of a free-standing $\mathrm{MoS}_{2}$ layer. The $\mathrm{MoS}_{2}$ wave functions strongly hybridize with those of the underlying Ti substrate, and the resulting hybridized states give a nonzero DOS for energies all through the $\mathrm{MoS}_{2}$ band gap. It is sometimes argued that such interface states promote having a good (Ohmic) $\mathrm{MoS}_{2} /$ metal contact $[19,21]$. One could, however, also argue that chemisorption is harmful to obtaining a good contact because it damages the integrity of the $\mathrm{MoS}_{2}$ layer. In Ref. [66], it is found that $\mathrm{MoS}_{2} / \mathrm{Ag}$ gives a better contact than $\mathrm{MoS}_{2} / \mathrm{Ti}$, due to a much smoother interface in the former case, suggesting to prefer physisorption over chemisorption.

It is not possible to define a Schottky barrier for a single $\mathrm{MoS}_{2}$ layer adsorbed on $\mathrm{Ti}(0001)$. Chemisorption affects the electronic structure of $\mathrm{MoS}_{2}$ to such an extent that its semiconducting character is lost. It is, however, possible to define a Schottky barrier for a second $\mathrm{MoS}_{2}$ layer that is adsorbed on the first layer. The first and second $\mathrm{MoS}_{2}$ layers are bonded by a van der Waals interaction, which does not perturb the electronic structure of the second layer significantly. Indeed, the DOS of the second $\mathrm{MoS}_{2}$ layer is quite similar to that of a free-standing $\mathrm{MoS}_{2}$ layer with a clear band gap (see Fig. 11). The height of the Schottky barrier to the second layer is a sizable $0.54 \mathrm{eV}$. This mainly results from the fact that the first adsorbed $\mathrm{MoS}_{2}$ layer effectively increases the work function of the Ti substrate (see Table VI). The size of the Schottky barrier indicates that it is not possible to make an Ohmic contact to pristine (undoped) $\mathrm{MoS}_{2}$ with Ti $[19,21]$.

\section{SUMMARY AND CONCLUSIONS}

In this paper, we explore the adsorption of $\mathrm{MoS}_{2}$ on a range of metal substrates by means of first-principles DFT calculations. The (111) surfaces of $\mathrm{Al}, \mathrm{Ni}, \mathrm{Cu}, \mathrm{Pd}, \mathrm{Ag}, \mathrm{Pt}$, and $\mathrm{Au}$ and the (0001) surfaces of $\mathrm{Mg}, \mathrm{Co}$, and $\mathrm{Ti}$ cover a range of metals with different interaction strengths, allowing for a systematic study of the metal-MoS $\mathrm{M}_{2}$ interface.

We compare the results obtained with different DFT functionals. In many cases, the GGA/PBE density functional only captures a small part of the binding energy of $\mathrm{MoS}_{2}$ on a metal substrate, as compared to the opt88-vdW-DF van der Waals density functional, which indicates the importance of van der Waals interactions in the interface bonding. Nevertheless, the equilibrium binding distances obtained with both functionals are generally very similar, and so are the interface potential steps and Schottky barrier heights. Exceptions are cases for which van der Waals interactions essentially describe the whole bonding, where PBE fails completely. LDA tends to overbind, leading to shorter binding distances and larger interface potential steps.

The interface structure that results from adsorbing an $\mathrm{MoS}_{2}$ layer on a metal surface will be incommensurable in most cases, as the two lattices have a mismatch. We investigate the effects of the artificial strain introduced by approximating the lattice using a commensurable supercell. We conclude that these effects are moderate provided the $\mathrm{MoS}_{2}$ lattice parameter is kept at its optimized value, and the metal lattice is strained. Large lattice mismatches should, however, be avoided, and straining the $\mathrm{MoS}_{2}$ lattice can lead to very unphysical results [19].

Of the metal substrates studied, $\mathrm{Ti}$ is the one on which $\mathrm{MoS}_{2}$ is clearly chemisorbed. Adsorption of $\mathrm{MoS}_{2}$ on $\mathrm{Ti}(0001)$ is accompanied by a clear structural deformation of the Ti surface and of the $\mathrm{MoS}_{2}$ overlayer, due to the formation of bonds between the surface $\mathrm{Ti}$ atoms and the sulfur atoms at the interface. Formation of these interface bonds significantly alters the electronic structure of the $\mathrm{MoS}_{2}$ adsorbate. In particular, the interface states fill up the band gap of $\mathrm{MoS}_{2}$, which makes defining a Schottky barrier for this layer meaningless. However, for a second, unperturbed, adsorbed $\mathrm{MoS}_{2}$ layer a Schottky barrier of $0.54 \mathrm{eV}$ can be extracted.

$\mathrm{MoS}_{2}$ is physisorbed on $\mathrm{Au}(111)$, where the bonding is almost completely due to van der Waals interactions, and the structure and electronic structure of $\mathrm{MoS}_{2}$ are hardly perturbed by the adsorption. The properties of $\mathrm{MoS}_{2}$ adsorbed on other metal substrates fall in the range between the two extreme cases ( $\mathrm{Ti}$ and $\mathrm{Au}$ ), without the possibility of drawing a clear dividing line, as has been done for the adsorption of graphene or $h$-BN on metal substrates $[14,15,24,26]$.

Experiments have focused foremost on Schottky barrier heights. Transport measurements on multilayer $\mathrm{MoS}_{2}$ devices generally yield small numbers for the Schottky barrier heights, i.e., $0.03-0.2 \mathrm{eV}$, for different metals $[7,8,11,20,67]$, whereas photoemission, photoconduction, and scanning tunneling spectroscopy give higher values $0.2-0.9 \mathrm{eV}[10,54,55,68]$. It has been suggested that the $\mathrm{MoS}_{2}$ samples used in devices are quite defective and inhomogeneous, such that the position of the Fermi level does not reflect an intrinsic property of $\mathrm{MoS}_{2}$ or of the $\mathrm{MoS}_{2} /$ metal contact [68,69], which obstructs a comparison to calculated results.

Our results for the $4 d$ and $5 d$ metals $\mathrm{Ag}, \mathrm{Au}, \mathrm{Pd}$, and $\mathrm{Pt}$ agree qualitatively with those reported in previous calculations [20-23], provided the $\mathrm{MoS}_{2}$ lattice is not stretched [19]. Quan- 
titatively, the reported Schottky barrier heights for these metals are $\sim 0.3 \mathrm{eV}$ larger than our results. These calculations were based upon the LDA functional, which tends to overbind, and to overestimate the metal work functions [14,26]. Compressing the metal lattice, which is sometimes required to accommodate a lattice mismatch in a small supercell, does not help either, as that gives an even higher work function [22]. The same is likely true for simple metals such as $\mathrm{Al}$ and In [21,22]. The strong interaction we find for $\mathrm{Ti}$ is also found in LDA calculations $[19,21,23]$. In those calculations, the lattice mismatch used was large, however, which can alter the interface interactions.

The overall picture emerging from these calculations is that $\mathrm{MoS}_{2}$ interacts strongly with the early transition metals, where it is clearly chemisorbed. The interaction with the late transition metals is much weaker, where the $3 d$ metals interact stronger than the $4 d$ and $5 d$ metals. $\mathrm{MoS}_{2}$ interacts rather weakly with the simple metals, but the interaction increases for very low-work-function metals. In all but the strongly chemisorbed case, van der Waals forces play an important role in the interface interactions.
In case the interface interaction is weak (physisorption), the interface potential step can be understood as resulting from Pauli repulsion, which effectively decreases the substrate work function. The Schottky barrier is then simply calculated from the modified work function. Strong interaction (chemisorption) leads to the formation of bonds between the substrate metal atoms and the adsorbate sulfur atoms. It increases the substrate work function if the electronegativity of the adsorbate is higher than that of the metal. If the $\mathrm{MoS}_{2}$ layer is chemisorbed, its electronic structure is perturbed to an extent that a Schottky barrier cannot be defined. However, a second adsorbed $\mathrm{MoS}_{2}$ layer then shows the characteristics of a single unperturbed layer.

\section{ACKNOWLEDGMENTS}

We acknowledge T. Amlaki and D. Çakır for fruitful discussions. This work is part of the research program of the Foundation for Fundamental Research on Matter (FOM), which is part of the Netherlands Organization for Scientific Research (NWO). The use of supercomputer facilities was sponsored by the Physical Sciences Division (EW) of NWO.
[1] M. Chhowalla, H. S. Shin, G. Eda, L.-J. Li, K. P. Loh, and H. Zhang, Nat. Chem. 5, 263 (2013).

[2] M. Xu, T. Liang, M. Shi, and H. Chen, Chem. Rev. 113, 3766 (2013).

[3] A. K. Geim and I. V. Grigorieva, Nature (London) 499, 419 (2013).

[4] K. F. Mak, C. Lee, J. Hone, J. Shan, and T. F. Heinz, Phys. Rev. Lett. 105, 136805 (2010).

[5] Q. H. Wang, K. Kalantar-Zadeh, A. Kis, J. N. Coleman, and M. S. Strano, Nat. Nanotechnol. 7, 699 (2012).

[6] D. Lembke, S. Bertolazzi, and A. Kis, Acc. Chem. Res. 48, 100 (2015).

[7] H. Liu, A. T. Neal, and P. D. Ye, ACS Nano 6, 8563 (2012).

[8] S. Das, H.-Y. Chen, A. V. Penumatcha, and J. Appenzeller, Nano Lett. 13, 100 (2013).

[9] J.-R. Chen, P. M. Odenthal, A. G. Swartz, G. C. Floyd, K. Y. L. Hua Wen, and R. K. Kawakami, Nano Lett. 13, 3106 (2013).

[10] M. Fontana, T. Deppe, A. K. Boyd, M. Rinzan, A. Y. Liu, M. Paranjape, and P. Barbara, Sci. Rep. 3, 113505 (2013).

[11] N. Kaushik, A. Nipane, F. Basheer, S. Dubey, S. Grover, M. M. Deshmukh, and S. Lodha, Appl. Phys. Lett. 105, 113505 (2014).

[12] J. Kang, W. Liu, and K. Banerjee, Appl. Phys. Lett. 104, 093106 (2014).

[13] M. Farmanbar and G. Brocks, Phys. Rev. B 91, 161304 (2015).

[14] M. Bokdam, G. Brocks, M. I. Katsnelson, and P. J. Kelly, Phys. Rev. B 90, 085415 (2014).

[15] M. Bokdam, G. Brocks, and P. J. Kelly, Phys. Rev. B 90, 201411 (2014).

[16] C. Ataca, H. Şahin, and S. Ciraci, J. Phys. Chem. C 116, 8983 (2012).

[17] W. A. Saidi, J. Chem. Phys. 141, 094707 (2014).

[18] J. P. Perdew and A. Zunger, Phys. Rev. B 23, 5048 (1981).

[19] I. Popov, G. Seifert, and D. Tománek, Phys. Rev. Lett. 108, 156802 (2012).
[20] W. Chen, E. J. G. Santos, W. Zhu, E. Kaxiras, and Z. Zhang, Nano Lett. 13, 509 (2013).

[21] J. Kang, W. Liu, D. Sarkar, D. Jena, and K. Banerjee, Phys. Rev. X 4, 031005 (2014).

[22] C. Gong, L. Colombo, R. M. Wallace, and K. Cho, Nano Lett. 14, 1714 (2014).

[23] Z. Li, X. Li, and J. Yang, ACS Appl. Mater. Interfaces 7, 12981 (2015).

[24] G. Giovannetti, P. A. Khomyakov, G. Brocks, V. M. Karpan, J. van den Brink, and P. J. Kelly, Phys. Rev. Lett. 101, 026803 (2008).

[25] R. Laskowski, P. Blaha, and K. Schwarz, Phys. Rev. B 78 045409 (2008).

[26] P. A. Khomyakov, G. Giovannetti, P. C. Rusu, G. Brocks, J. van den Brink, and P. J. Kelly, Phys. Rev. B 79, 195425 (2009).

[27] D. Stradi, S. Barja, C. Díaz, M. Garnica, B. Borca, J. J. Hinarejos, D. Sánchez-Portal, M. Alcamí, A. Arnau, A. L. Vázquez de Parga, R. Miranda, and F. Martín, Phys. Rev. Lett. 106, 186102 (2011).

[28] T. Olsen, J. Yan, J. J. Mortensen, and K. S. Thygesen, Phys. Rev. Lett. 107, 156401 (2011).

[29] M. Andersen, L. Hornekær, and B. Hammer, Phys. Rev. B 86, 085405 (2012)

[30] P. Janthon, F. Viñes, S. M. Kozlov, J. Limtrakul, and F. Illas, J. Chem. Phys. 138, 244701 (2013).

[31] J. P. Perdew, K. Burke, and M. Ernzerhof, Phys. Rev. Lett. 77, 3865 (1996).

[32] D. Çakır, C. Sevik, and F. M. Peeters, J. Mater. Chem. C 2, 9842 (2014).

[33] D. Çakır and F. M. Peeters, Phys. Rev. B 89, 245403 (2014).

[34] B. Sachs, T. O. Wehling, M. I. Katsnelson, and A. I. Lichtenstein, Phys. Rev. B 84, 195414 (2011).

[35] E. Hazrati, G. A. de Wijs, and G. Brocks, Phys. Rev. B 90, 155448 (2014). 
[36] M. Dion, H. Rydberg, E. Schröder, D. C. Langreth, and B. I. Lundqvist, Phys. Rev. Lett. 92, 246401 (2004).

[37] T. Thonhauser, V. R. Cooper, S. Li, A. Puzder, P. Hyldgaard, and D. C. Langreth, Phys. Rev. B 76, 125112 (2007).

[38] J. Klimeš, D. R. Bowler, and A. Michaelides, Phys. Rev. B 83, 195131 (2011).

[39] G. Kresse and J. Hafner, Phys. Rev. B 47, 558 (1993).

[40] P. E. Blöchl, Phys. Rev. B 50, 17953 (1994).

[41] G. Kresse and J. Furthmüller, Phys. Rev. B 54, 11169 (1996).

[42] G. Kresse and D. Joubert, Phys. Rev. B 59, 1758 (1999).

[43] M. Methfessel and A. T. Paxton, Phys. Rev. B 40, 3616 (1989).

[44] J. Neugebauer and M. Scheffler, Phys. Rev. B 46, 16067 (1992).

[45] G. Giovannetti, P. A. Khomyakov, G. Brocks, P. J. Kelly, and J. van den Brink, Phys. Rev. B 76, 073103 (2007).

[46] T. Björkman, A. Gulans, A. V. Krasheninnikov, and R. M. Nieminen, Phys. Rev. Lett. 108, 235502 (2012).

[47] I. Hamada, Phys. Rev. B 89, 121103 (2014).

[48] H. Shi, H. Pan, Y.-W. Zhang, and B. I. Yakobson, Phys. Rev. B 87, 155304 (2013).

[49] T. Li, Phys. Rev. B 85, 235407 (2012).

[50] W. S. Yun, S. W. Han, S. C. Hong, I. G. Kim, and J. D. Lee, Phys. Rev. B 85, 033305 (2012).

[51] E. Cappelluti, R. Roldán, J. A. Silva-Guillén, P. Ordejón, and F. Guinea, Phys. Rev. B 88, 075409 (2013).

[52] F. Hüser, T. Olsen, and K. S. Thygesen, Phys. Rev. B 88, 245309 (2013).

[53] H. J. Conley, B. Wang, J. I. Ziegler, J. Richard F. Haglund, S. T. Pantelides, and K. I. Bolotin, Nano Lett. 13, 3626 (2013).

[54] J. R. Lince, D. J. Carré, and P. D. Fleischauer, Phys. Rev. B 36, 1647 (1987).

[55] C. Maurel, F. Ajustron, R. Péchou, G. Seine, and R. Coratger, Surf. Sci. 600, 442 (2006).
[56] P. A. Young, J. Phys. D Appl. Phys. 1, 936 (1968).

[57] A. Al-Hilli and B. Evans, J. Cryst. Growth 15, 93 (1972).

[58] T. Böker, R. Severin, A. Müller, C. Janowitz, R. Manzke, D. Voß, P. Krüger, A. Mazur, and J. Pollmann, Phys. Rev. B 64, 235305 (2001).

[59] H.-P. Komsa and A. V. Krasheninnikov, Phys. Rev. B 88, 085318 (2013).

[60] C. Gong, G. Lee, B. Shan, E. M. Vogel, R. M. Wallace, and K. Cho, J. Appl. Phys. 108, 123711 (2010).

[61] S. Tongay, J. Zhou, C. Ataca, K. Lo, T. S. Matthews, J. Li, J. C. Grossman, and J. Wu, Nano Lett. 12, 5576 (2012).

[62] M. M. Ugeda, A. J. Bradley, S.-F. Shi, F. H. da Jornada, Y. Zhang, D. Y. Qiu, W. Ruan, S.-K. Mo, Z. Hussain, Z.-X. Shen, F. Wang, S. G. Louie, and M. F. Crommie, Nat. Mater. 13, 1091 (2014).

[63] J.-W. van der Horst, P. A. Bobbert, M. A. J. Michels, G. Brocks, and P. J. Kelly, Phys. Rev. Lett. 83, 4413 (1999).

[64] W. S. Leong, X. Luo, Y. Li, K. H. Khoo, S. Y. Quek, and J. T. L. Thong, ACS Nano 9, 869 (2015).

[65] Y. Du, L. Yang, J. Zhang, H. Liu, K. Majumdar, P. Kirsch, and P. Ye, IEEE Electron Device Lett. 35, 599 (2014).

[66] H. Yuan, G. Cheng, L. You, H. Li, H. Zhu, W. Li, J. J. Kopanski, Y. S. Obeng, A. R. H. Walker, D. J. Gundlach, C. A. Richter, D. E. Ioannou, and Q. Li, ACS Appl. Mater. Interfaces 7, 1180 (2015).

[67] H. Qiu, L. Pan, Z. Yao, J. Li, Y. Shi, and X. Wang, Appl. Phys. Lett. 100, 123104 (2012).

[68] S. McDonnell, R. Addou, C. Buie, R. M. Wallace, and C. L. Hinkle, ACS Nano 8, 2880 (2014).

[69] M. Yankowitz, S. Larentis, K. Kim, J. Xue, D. McKenzie, S. Huang, M. Paggen, M. N. Ali, R. J. Cava, E. Tutuc, and B. J. LeRoy, Nano Lett. 15, 1925 (2015). 